\title{
Evidence for risk stratification when monitoring for toxicities following initiation of combination antiretroviral therapy
}

\author{
Babafemi Taiwo $^{\mathrm{a}}$, Elizabeth L. Yanik ${ }^{\mathrm{b}}$, Sonia Napravnik ${ }^{\mathrm{b}}$, Patrick Ryscavage ${ }^{\mathrm{c}}$, Susan L. \\ Koletar $^{d}$, Richard Moore ${ }^{e}$, W. Christopher Mathews ${ }^{\dagger}$, Heidi M. Crane ${ }^{g}$, Kenneth Mayer ${ }^{h}$, \\ Anne Zinski', James S. Kahn', Joseph J. Eron ${ }^{b}$, and on behalf of the CFAR Network of \\ Integrated Clinical Systems (CNICS) Cohort Study
}

aNorthwestern University, Chicago, Illinois bUniversity of North Carolina, Chapel Hill, North Carolina 'University of Maryland, Baltimore, Maryland dOhio State University, Columbus, Ohio e Johns Hopkins University, Baltimore, Maryland fUniversity of California, San Diego, California gUniversity of Washington, Seattle, Washington hBeth Israel Deaconess Medical Center, Boston, Massachusetts 'University of Alabama, Birmingham, Alabama jVeterans Administration Palo Alto Healthcare System, Palo Alto, California, USA

\begin{abstract}
Objective-Laboratory monitoring is recommended during combination antiretroviral therapy (cART), but the pattern of detected abnormalities and optimal monitoring are unknown. We assessed laboratory abnormalities during initial cART in 2000-2010 across the United States.
\end{abstract}

Design-Observational study in the Centers for AIDS Research Network of Integrated Clinical Systems Cohort.

\begin{abstract}
Methods-Among patients with normal results within a year prior to cART initiation, time to first significant abnormality was assessed by Kaplan-Meier curves stratified by event type, with censoring at first of regimen change, loss to follow-up, or 104 weeks. Incidence rates of first events were estimated using Poisson regression; multivariable analyses identified associated factors. Results were stratified by time (16 weeks) from therapy initiation.
\end{abstract}

Results-A total of 3470 individuals contributed 3639 person-years. Median age, pre-cART CD4, and follow-up duration were 40 years, 206 cells/ $\mu$ l, and 51 weeks, respectively. Incidence rates for significant abnormalities (per 100 person-years) in the first 16 weeks post-cART initiation were as follows: lipid 49 [95\% confidence interval (CI) 41- 58]; hematologic $=44$ (4049); hepatic $=24(20-27)$; and renal $=9(7-11)$, dropping substantially during weeks 17-104 of cART to lipid = $23(18-29)$; hematologic $=5(4-6)$; hepatic $=6(5-8) ;$ and renal $=2(1-3)($ all $P<$

(C) 2013 Wolters Kluwer Health | Lippincott Williams \& Wilkins

Correspondence to Babafemi Taiwo, MBBS, Infectious Diseases Division, Northwestern University, 645 N Michigan Avenue, Chicago, IL 60611, USA. Tel: +1 312695 5085; b-taiwo@ northwestern.edu.

Conflicts of interest

B.T. has served as on advisory boards of ViiV, Pfizer, GSK, Gilead, and received research support (to Northwestern University) from Pfizer. R.M. has received research grants from Pfizer. J.J.E is a consultant to Abbott, GlaxoSmithKline, Merck, GSK/ViiV, Janssen, and Gilead, and has received research support (to UNC) from GlaxoSmithKline, Merck, and GSK/ViiV. The other authors report no conflicts of interest. 
0.05). Among patients receiving initial cART with no prior abnormality $(N=1889)$, strongest associations for hepatic abnormalities after 16 weeks were hepatitis $\mathrm{B}$ and $\mathrm{C}$ [hazard ratio 2.3 $(95 \%$ CI 1.2-4.5) and hazard ratio $=3.0(1.9-4.5)$, respectively]. The strongest association for renal abnormalities was hypertension [hazard ratio $=2.8(1.4-5.6)]$.

Conclusion-New abnormalities decreased after week 16 of cART. For abnormalities not present by week 16, subsequent monitoring should be guided by comorbidities.

\section{Keywords}

hematologic; hepatic; laboratory; lipid; monitoring HIV; renal

\section{Introduction}

Adverse effects of combination antiretroviral therapy (cART) range from asymptomatic laboratory abnormalities to life-threatening events. Although guidelines exist in the United States for laboratory monitoring during cART [1], the optimal monitoring schedule to balance patient safety and rational utilization of resources has not been determined. This balance is necessary as healthcare resources are shrinking [2,3] while the number of patients receiving cART is expected to increase as a result of new infections [4], intensified efforts to identify and retain HIV-infected patients in care [5], and recommendation of cART in all HIV-infected patients [6]. Further, studies in resource-limited settings have found no clinical benefit to routine laboratoring monitoring for adverse effects during cART [7,8], possibly with the exception of hemoglobin monitoring in those taking zidovudine (ZDV) [7].

HIV-infected persons are a heterogeneous population and a variety of antiretroviral drug combinations are approved for initial cART [1]. Although it has been suggested that patient and regimen differences may influence risk of specific adverse events [9-13], current monitoring guidelines in the United States are largely the same regardless of patient populations and regimens. This approach may lead to unnecessary monitoring for uncommon adverse events in low-risk patients or inadequate monitoring for other adverse events in patients with higher risk. Understanding the incidence and predictors of significant laboratory abnormalities (i.e. those that require cART modification, further evaluation, or direct treatment) is necessary to define optimal monitoring practices. We conducted this study to determine the incidence and risk factors for significant hematologic, hepatic, renal, and lipid abnormalities in patients who initiated cART in routine clinical care in the United States between 2000 and 2010 .

\section{Methods}

\section{Study setting}

This observational study was conducted using data from the Centers for AIDS Research (CFAR) Network of Integrated Clinical Systems (CNICS) cohort. CNICS is a longitudinal clinical cohort of HIV-infected patients receiving primary care from 1 January 1995 to the present at eight medical centers across the United States, all affiliated with CFAR. The CNICS cohort is diverse with regard to sex, race, ethnicity, age, HIV transmission risk factors, and geographic distribution. The CNICS repository contains data from electronic 
medical record systems used to provide HIV care, and support clinical and translational HIV research at the affiliated clinics [14].

\section{Study population}

The study population consisted of HIV-infected patients who were at least 18 years old; initiated cARTat a CNICS site between 1 January 2000 and 31 January 2010; had a normal result for their most recent laboratory test within a year prior to cART initiation based on the definition of abnormalities used to evaluate outcomes; and had at least one laboratory monitoring test result available after cART initiation while still receiving their initial cART regimen. We considered hematologic, hepatic, renal, and lipid laboratory values post-cART initiation and defined significant abnormalities as follows: hematologic $=$ hemoglobin 10 $\mathrm{g} / \mathrm{dl}$ or less, neutrophil count 750 cells/ $/ \mathrm{l}$ or less, or platelet count $50 \times 10^{9}$ cells/l or less; hepatic $=$ aspartate or alanine transaminase at least three times the upper limit of normal (ULN) or direct bilirubin at least two times ULN; renal = estimated glomerular filtration rate (eGFR) less than $50 \mathrm{ml} / \mathrm{min}$ per $1.73 \mathrm{~m}^{2}$. eGFR was calculated using the Modification of Diet in Renal Disease (MDRD) formula [15]; a cut-off of less than $60 \mathrm{ml} / \mathrm{min}$ per $1.73 \mathrm{~m}^{2}$ was used in sensitivity analysis; and lipid abnormality = non-high-density lipo-protein (HDL) cholesterol (i.e. total cholesterol minus HDL cholesterol) at least $160 \mathrm{mg} / \mathrm{dl}$. Ethical approval for CNICS was obtained from each site's Institutional Review Board, and for this study from the University of North Carolina Institutional Review Board.

\section{Combination antiretroviral therapy definition}

To ensure that only regimens relevant to initial therapy in the modern cARTera were considered, we defined cART as a regimen containing a ritonavir-boosted protease inhibitor, nonnucleoside reverse transcriptase inhibitor (NNRTI), or integrase inhibitor along with at least two nucleos(t)ide reverse transcriptase inhibitors (NRTIs). Patients whose regimen included didanosine, stavudine, nelfinavir, indinavir, unboosted protease inhibitor, or more than two antiretroviral classes were excluded.

\section{Statistical analysis}

At-risk time for each patient began at cART initiation and ended at the first abnormality for the laboratory index being evaluated, switch or discontinuation (for $>14$ days) of any drug in the regimen, loss to follow-up ( $\geq 12$ months without a clinic visit or laboratory measure), last clinic visit before 31 December 2010, or death. Follow-up was censored at 104 weeks after cART initiation to ensure sufficient at-risk patients to obtain reliable estimates of abnormality rates. Baseline characteristics at cART initiation were described and time to first abnormality was estimated by the Kaplan-Meier method. Time to first abnormality was calculated for each abnormality type. Overall incidence rates of first abnormalities and incidence rates stratified by follow-up time were estimated using Poisson regression.

Hazard ratios were estimated to identify independent clinical and demographic predictors of an incident laboratory abnormality using multivariable pooled logistic regression and inverse-probability-of-frequency weights to account for differences in laboratory monitoring frequency $[16,17]$. These weights were calculated using predicted probabilities of receiving a laboratory test conditional on baseline covariates. The means for the weights were 1.09 for 
hematologic [standard deviation $(\mathrm{SD})=0.34$, full range $($ range $)=0.33-2.50$ ], 1.11 for hepatic $(\mathrm{SD}=0.36$, range $=0.29-2.60), 1.12$ for renal $(\mathrm{SD}=0.37$, range $=0.29-2.84)$, and 1.16 for lipid $(\mathrm{SD}=0.53$, range $=0.35-3.95)$. Multivariable analyses included all demographic and clinical variables being evaluated as predictors as well as study site.

We evaluated the effect of time since cART initiation on the incidence of each laboratory abnormality. Given substantial heterogeneity upon visual inspection of incidence rates across time and formal statistical evaluation of changes in incidence rates, we report results stratified by time from therapy initiation (0-16 and 17-104 weeks). For analyses evaluating rates from 17- 104 weeks of cARTexposure by laboratory type, we only included patients who remained on their initial cART through 16 weeks of therapy; and had at least one normal value, and no abnormal values, for that laboratory type between 0 and 16 weeks post-cART. In order to obtain measures of frequency for patients at differing levels of risk after 16 weeks, Poisson regression was used to calculate incidence rates between 17 and 104 weeks within subgroups of patients defined by the strongest clinically meaningful predictors identified in multivariable analyses. All analyses were conducted in SAS version 9.2 (SAS Institute Inc., Cary, North Carolina, USA).

\section{Results}

\section{Study population}

The study population included 3470 patients who had at least one laboratory result following cART initiation, including 3153, 3219, 3208, and 980 with at least one hematologic, hepatic, renal, and lipid test, respectively. Patients without available pre-cART laboratory measures or with documented pre-cART laboratory abnormalities were excluded (Fig. 1). The population was $17.1 \%$ women, $36.3 \%$ black, and $43.8 \%$ white with a median age of 40 years [interquartile range (IQR) 32-45] (Table 1). The median year of cART initiation was 2006 (IQR 2004-2008). At therapy initiation, the median CD4 cell count was 206 cells/ $\mu \mathrm{l}$ (IQR 65-316) and the median $\log _{10}$ viral load was 4.9 copies/ml (IQR 4.4-5.4). The initial regimens included an NNRTI (62.2\%), mainly efavirenz (95.9\% of NNRTIs); or a boosted protease inhibitor $(37.5 \%)$, most commonly atazanavir/ritonavir (56.0\% of protease inhibitors) or lopinavir/ritonavir (35.9\% of protease inhibitors). Patient demographic and clinical characteristics at cART initiation were similar across evaluated laboratory abnormalities and follow-up time contributed.

The median time under observation was 51 weeks (IQR 19-104), yielding a total of 3639 person-years observed. Twenty-seven percent of patients remained on their first cART and under observation for the full 104 weeks of follow-up, 34.4\% changed their initial regimen or had a regimen interruption, $18.3 \%$ were lost to follow-up, and $21.8 \%$ were censored on 31 December 2010 when follow-up ended. Patients had a median of 11 (IQR 5-18) hematologic, nine (4-16) hepatic, four (2-7) renal, and two (1-4) lipid tests while on their initial cART regimen. 


\section{Incidence of first significant laboratory abnormalities}

Over 104 weeks, a total of 867 patients experienced at least one hematologic, hepatic, renal, or lipid abnormality, yielding an overall incidence rate of 33.8 per 100 person-years [95\% confidence interval (CI) 31.6-36.1]. Hematologic abnormalities (incidence rate of 15.2 per 100 person-years; 95\% CI 13.9-16.7) were largely due to low hemoglobin (64.8\% of total abnormalities). The incidence rate for a hepatic abnormality was 11.0 per 100 person-years (95\% CI 9.9-12.2) and was driven by abnormal transaminases (75.8\%). The incidence rate for eGFR decline to less than $50 \mathrm{ml} / \mathrm{min}$ was 3.5 per 100 person-years (95\% CI 2.9-4.3). When we used an eGFR cut-off of less than $60 \mathrm{ml} / \mathrm{min}$, the incidence rate was 7.8 (95\% CI 6.8-8.8). Lipid abnormalities were the most frequent with an incidence rate of 32.1 per 100 person-years (95\% CI 28.7-36.1; Table 2).

The cumulative probability of experiencing a laboratory abnormality by 104 weeks of treatment was $17.7 \%$ for hematologic, $15.2 \%$ for hepatic, $5.1 \%$ for renal, and $41.9 \%$ for lipid indices. Figure 2 illustrates that abnormalities were most common within the first 16 weeks. As evident in Table 2, this elevated risk soon after cART initiation is particularly notable for hematologic abnormalities, which were nine times more common in the first 16 weeks of therapy compared with weeks $17-104(P<0.001)$. Hepatic and renal events were four and five times more frequent pre versus postweek 16 of therapy, respectively (both $P<0.001$ ). The drop in incidence rate after week 16 was less striking for lipid abnormalities, which were approximately twice as frequent in the first 16 weeks $(P<0.001)$.

\section{Predictors of first significant laboratory abnormalities}

Female sex, African-American race, hepatitis B coin-fection, hypertension, boosted protease inhibitor use, ZDV use, lower CD4 cell count, and AIDS diagnosis at cART initiation were all independently predictive of a first hematologic abnormality during the first 104 weeks of cART in multivariable analyses (Table 3). Analogous results were observed after restricting to events occurring during the first 16 weeks of cARTuse. After 16 weeks of cART (among those with no hematologic abnormality in first 16 weeks), boosted protease inhibitor use was associated with a 1.8 -fold increase in the incidence rate. $\mathrm{CD}^{+} \mathrm{T}$-cell reconstitution during cART predicted a lower hematologic abnormality rate; for each $50 \mathrm{CD} 4$ cells/ $\mu \mathrm{l}$ increase from baseline to 16 weeks, there was an $11.5 \%$ relative reduction in the incidence rate. These associations were not modified by ZDV use.

Lower CD4 cell count and AIDS diagnosis at cART initiation, other race (which included Hispanic, Asian, American-Indian, and multiracial), boosted protease inhibitor use, and coinfection with hepatitis $\mathrm{B}$ or $\mathrm{C}$ were associated with a higher rate of hepatic abnormality during the total period of observation. Findings were similar when we considered the first 16 weeks of cART only. During weeks 17-104 of cART, hepatitis coinfections were most strongly associated with hepatic abnormality with hepatitis B associated with more than twice the rate of abnormalities, and hepatitis $\mathrm{C}$ associated with almost three times the rate (Table 3). Boosted protease inhibitor use was associated with a 1.75 -fold increase (95\% CI 1.20-2.56). Next, we evaluated the absolute impact of these predictors on hepatic abnormality rates. Patients coinfected with hepatitis B or C consistently experienced more hepatic abnormalities; for example, 16 additional abnormalities per 100 person-years 
between 17 and 104 weeks of cART when compared to those without hepatitis B or C coinfection (Table 4). Among patients with hepatitis coinfection, the rates of hepatic abnormalities before and after week 16 were higher among those treated with boosted protease inhibitors.

Decline in eGFR to less than $50 \mathrm{ml} / \mathrm{min}$ per $1.73 \mathrm{~m}^{2}$ during the first 104 weeks of cART was most strongly associated with preexisting hypertension (Table 3 ). Other risk factors were female sex, older age, lower CD4 cell counts, and boosted protease inhibitor use. Risk factors were the same when restricting analyses to the first 16 weeks of cART. After 16 weeks, the strongest predictors of renal function decline were hypertension, female sex, and greater increase in CD4 cell count. However, after 16 weeks, incidence rates were low even in higher risk groups. For instance, patients with hypertension on a boosted protease inhibitor-based regimen had an incidence rate of 5.9 (95\% CI 3.4-10.3; Table 4). When we changed the definition of significant renal abnormality to eGFR less than $60 \mathrm{ml} / \mathrm{min}$ per 1.73 $\mathrm{m}^{2}$, similar associations after 16 weeks were found, although older age and boosted protease inhibitor use became the strongest predictors. African-American race was identified as an additional independent predictor over the 104-week period using the less than $60 \mathrm{ml} / \mathrm{min}$ per $1.73 \mathrm{~m}^{2}$ cut-off (data not shown).

Lipid abnormalities within the first 16 weeks of cART were more common among patients who were older, men, of non-African-American race, had an AIDS-defining illness, or were hepatitis B and C negative. After 16 weeks post-cART initiation, no statistically significant associations were identified (Table 3).

\section{Discussion}

We found that first significant hematologic, hepatic, renal, and lipid laboratory abnormalities were most common in the early period (first 16 weeks) after initiating cART. Among patients who remained on their initial regimen without abnormalities by week 16 , the incidence of subsequent laboratory abnormalities decreased substantially. Abnormalities that occurred after week 16 (delayed abnormalities) were associated with pretreatment comorbidities and cART regimen. Thus, hepatitis B or C coinfection were associated with increased risk for delayed hepatic abnormalities, whereas hypertension was associated with increased risk for delayed renal impairment. Compared to NNRTIs, boosted protease inhibitor use was associated with increased risk of delayed hematologic, hepatic, and renal abnormalities.

Although current guidelines recommend laboratory monitoring within 2-8 weeks of initiating cART and then every 3-6 months afterwards in all patients [1], our findings suggest that indefinite monitoring every 3-6 months without stratification by risk may not be necessary in all patients. For example, long-term hepatic monitoring every 3-6 months may be appropriate for hepatitis-coinfected patients because hepatitis $\mathrm{B}$ virus is associated with over two-fold increased risk for delayed hepatic abnormalities and hepatitis $\mathrm{C}$ virus (HCV) with approximately three-fold increased risk. In contrast, patients at relatively low risk for delayed hepatic abnormalities (e.g. those not infected with hepatitis B or C) should be considered for less frequent monitoring. We observed a low incidence of new renal 
abnormalities after 16 weeks, particularly in patients younger than 60 years without hypertension, indicating that individuals in this subgroup may be candidates for less frequent renal monitoring, although renal abnormalities may increase with longer duration of therapy.

Our findings are strengthened by the fact that many of the parameters that we identified as risk factors for laboratory abnormalities are well established. A recent study noted higher rates of hepatotoxicity among hepatitis $\mathrm{B}$ and/or $\mathrm{C}$-coinfected patients receiving boosted protease inhibitor regimens compared to those taking efavirenz-based regimens [18], a finding corroborated in our study. Hypertension, a risk factor for renal abnormalities in the current study, is a well known predictor of chronic kidney disease in both HIV-infected and HIV-uninfected populations [19,20]. The hematologic side-effect profile of ZDV was well described by the early 1990s [21], although a more recent study suggested a weaker link between ZDV and anemia in the cART era [22]. In our study, ZDV use was associated with hematologic abnormalities, but this effect extended only through the first 16 weeks of therapy suggesting that ZDV use alone may not justify intensive hematologic monitoring indefinitely. The observed higher rates of anemia among women and neutropenia in African-Americans likely reflect lower baseline values in these subgroups [23,24]. Lipid abnormalities were most common in the first 16 weeks in our study, which is consistent with a large CNICS study that observed the greatest increase in lipid levels during the first 8 weeks of cART [25]. Of note, however, patients who have lipid levels measured within 4 months of therapy may represent a specific subpopulation that clinicians expect to have higher risk for lipid abnormalities. Boosted protease inhibitor use was associated with renal abnormalities, although an independent effect of tenofovir exposure on renal impairment was not identified when we used an eGFR cut-off of $50 \mathrm{ml} / \mathrm{min}$ per $1.73 \mathrm{~m}^{2}$. It is possible that detection of a tenofovir-specific effect on eGFR decline below $50 \mathrm{ml} / \mathrm{min}$ per $1.73 \mathrm{~m}^{2}$ was hampered by the high prevalence of tenofovir use $(73 \%)$. There are varying reports of the associations between tenofovir and renal toxicity [10,26-30] and concomitant boosted protease inhibitor exposure appears to heighten tenofovir toxicity risks [31]. There was an unexpected independent association between boosted protease inhibitor use and hematologic abnormalities, and an explanation for this association is not readily apparent.

This study is limited by the laboratory measurements available. Specifically, timing of the measurements was variable given the real-world clinical care setting in which they were obtained. However, we mitigated this limitation by incorporating inverse-probability-offrequency weights into the analysis to reduce the influence of patients who were tested more frequently. Further, we did not have comprehensive data on HCV status of seropositive patients; hence, some patients labeled with HCV infection on the basis of serologic testing could have subsequently cleared their infection. This could have affected the precision of the estimated impact of HCV. Also, although current guidelines recommend fasting lipids, random lipid levels were obtained in many of patients; hence, we focused on non-HDL cholesterol. Non-HDL cholesterol levels have an increasingly important role in cardiovascular disease risk assessment and lipid-lowering treatment decisions [32], particularly in the setting of elevated triglyceride levels, which is common among HIVinfected individuals. 
Other factors should be considered when interpreting our study. First, only $36 \%$ of patients evaluated in our study were in care and maintained on their initial cART regimen at week 104, reflecting challenges of patient retention [33] and switching to different regimens for a variety of reasons. Further research will be needed to evaluate whether the associations we identified extend beyond 2 years. Second, because we excluded patients with baseline abnormalities for the laboratory type being evaluated and censored patient data after the first laboratory abnormality of that type, this study does not address laboratory abnormalities after an abnormal index value. Third, although our findings suggest that ritonavir-boosted protease inhibitors may be associated with some increase in laboratory abnormalities when compared to NNRTI-based regimens, this study was not designed to specifically assess comparative safety of different regimens. We were unable to comprehensively address issues such as dose of ritonavir, other agents (both antiretrovirals and non-antiretrovirals coadministered), preexisting nonsignificant elevations in laboratory measures, and factors that may predispose patients to receiving one type of cART regimen versus another. The observational design of our study may have introduced other unmeasured confounding, but we were able to utilize longitudinal comprehensive clinical data from a large number of patients in routine care at diverse sites across the United States, making our results generalizable. Frequently, results from randomized trials cannot be generalized to patients in routine clinical care because of extensive inclusion and exclusion criteria that result in enrolling individuals who may have a lower likelihood of adverse events [34]. Women represented only $17 \%$ of the total population, the median duration of follow-up was relatively short (51 weeks), and median pre-cART CD4 cell count was low (206 cells/ $\mu \mathrm{l})$. Furthermore, only one measurement was available post-cART initiation for a proportion of patients. Finally, there is increasing representation of persons older than 45 years in the HIV population [4], a group at increased risk for age-associated noninfectious comorbidities and polypharmacy [35]. A quarter of the patients in our study were over 45 years at ART initiation, although the median age was 40 years.

\section{Conclusion}

In conclusion, among patients with normal laboratory indices at cART initiation, monitoring for early laboratory abnormalities while asymptomatic appears justifiable in all patients initiating CART. However, our findings suggest that long-term monitoring should vary between patients and be informed by comorbidities and possibly cART regimen. If our preliminary findings are confirmed and risk-guided monitoring is adopted, it would represent a paradigm shift in the care of US HIV-infected patients with implications for lifetime treatment costs and utilization of healthcare resources. Further work is indicated that specifically focuses on comparative safety across regimens that are currently recommended for initial cART. Future studies should also refine the parameters for risk stratification, the optimal frequency of testing in high-risk and low-risk subgroups, and the cost-effectiveness of risk-guided monitoring.

\section{Acknowledgments}

B.T., E.L.Y., and S.N. led the conception and design of this study, contributed to data acquisition and interpretation, prepared the initial draft of the article, had full access to all the data in the study, and take final responsibility for the decision to submit for publication. J.J.E., P.R., and S.L.K. substantively contributed to the conception and design of 
this study, data acquisition and/or interpretation, and provided critical revision of the article. Remaining authors provided substantial contributions to the study design, data acquisition, and/or interpretation of data and provided critical revision of the article. All authors approved the final version of the article.

The authors would like to thank the patients, principal investigators, coinvestigators, and research staff at participating Centers for AIDS Research Network of Integrated Clinical Systems sites at the following institutions: Case Western Reserve University; University of Alabama at Birmingham; University of California at San Francisco; University of Washington; University of California at San Diego; Fenway Community Health Center of Harvard University; University of North Carolina at Chapel Hill; and Johns Hopkins University. In particular, they thank Stephen Van Rompaey of the Data Management Core at University of Washington and Donna Porter of the Administrative Core at University of Alabama at Birmingham for their assistance.

This work was supported by grants from the National Institutes of Health (R24-AI067039, P30-AI50410, and 5T32AI007001-35); and the Agency for Healthcare Research and Quality (R01-HS018731). The funding sources did not participate in the study design; collection, analysis and interpretation of data; in the writing of the article; or in the decision to submit the paper for publication.

\section{References}

1. Panel on Antiretroviral Guidelines for Adults and Adolescents. Guidelines for the use of antiretroviral agents in HIV-1-infected adults and adolescents. Department of Health and Human Services; Mar 27. 2012 p. 1-240.http://aidsinfo.nih.gov/contentfiles/AdultandAdolescentGL.pdf [29 March 2012]

2. Bassett IV, Farel C, Szmuilowicz ED, Walensky RP. HIV/AIDS: AIDS Drug Assistance Programs in the era of routine HIV testing. Clin Infect Dis. 2008; 47:695-701. [PubMed: 18643759]

3. Snyder, L. Ann Intern Med. 6th ed.. Vol. 156. American College of Physicians Ethics Manual; 2012. American College of Physicians Ethics, Professionalism, and Human Rights Committee.; p. 73-104.

4. Centers for Disease Control. HIV surveillance - United States, 1981-2008. MMWR. 2011; 60:689_ 693. [PubMed: 21637182]

5. The White House Office of National AIDS Policy. National HIV/ AIDS strategy for the United States. White House; Washington DC: 2010. http://aids.gov/federal-resources/national-hiv-aidsstrategy/nhas.pdf. [1 September 2012]

6. Thompson MA, Aberg JA, Hoy JF, Telenti A, Benson C, Cahn P, et al. Antiretroviral treatment of adult HIV infection: 2012 Recommendations of the International Antiviral Society-USA Panel. JAMA. 2012; 308:387-402. [PubMed: 22820792]

7. Koenig SP, Schackman BR, Riviere C, Leger P, Charles M, Severe P, et al. Clinical impact and cost of monitoring for asymptomatic laboratory abnormalities among patients receiving anti-retroviral therapy in a resource-poor setting. Clin Infect Dis. 2010; 51:600-608. [PubMed: 20649436]

8. Mugyenyi P, Walker AS, Hakim J, Munderi P, Gibb DM, Kityo C, et al. Routine versus clinically driven laboratory monitoring of HIV antiretroviral therapy in Africa (DART): a randomised noninferiority trial. Lancet. 2010; 375:123-131. [PubMed: 20004464]

9. Blanco JR, Caro AM, Pérez-Cachafeiro S, Gutiérrez F, Iribarren JA, González-García J, et al. HIV infection and aging. AIDS Rev. 2010; 12:218-230. [PubMed: 21179186]

10. Tordato F, Cozzi Lepri A, Cicconi P, De Luca A, Antinori A, Colangeli V, et al. Evaluation of glomerular filtration rate in HIV-1-infected patients before and after combined antiretroviral therapy exposure. HIV Med. 2011; 12:4-13. [PubMed: 20584091]

11. Towner WJ, Xu L, Leyden WA, Horberg MA, Chao CR, Tang B, et al. The effect of HIV infection, immunodeficiency and antiretroviral therapy on the risk of hepatic dysfunction. $\mathrm{J}$ Acquir Immune Defic Syndr. 2012; 60:321-327. [PubMed: 22343179]

12. Gallant JE, DeJesus E, Arribas JR, Pozniak AL, Gazzard B, Campo RE, et al. Tenofovir DF, emtricitabine, and efavirenz vs. zidovudine, lamivudine, and efavirenz for HIV. N Engl J Med. 2006; 354:251-260. [PubMed: 16421366]

13. Sierra-Madero J, Villasis-Keever A, Méndez P, Mosqueda-Gómez JL, Torres-Escobar I, GutierrezEscoloano F, et al. Prospective, randomized, open label trial of efavirenz vs lopinavir/ ritonavir in HIVR treatment-naive subjects with CD4R $<200 \mathrm{cell} / \mathrm{mm}^{3}$ in Mexico. J Acquir Immune Defic Syndr. 2010; 53:582-588. [PubMed: 20090545] 
14. Kitahata MM, Rodriguez B, Haubrich R, Boswell S, Mathews WC. Cohort profile: the Centers for AIDS Research Network of Integrated Clinical Systems. Int J Epidemiol. 2008; 37:948-955. [PubMed: 18263650]

15. Levey AS, Bosch JP, Lewis JB, Greene T, Rogers N, Roth D. A more accurate method to estimate glomerular filtration rate from serum creatinine: a new prediction equation. Ann Intern Med. 1999; 130:461-470. [PubMed: 10075613]

16. Hernan MA, McAdams M, McGrath N, Lanoy E, Costagliola D. Observation plans in longitudinal studies with time-varying treatments. Stat Methods Med Res. 2009; 18:27-52. [PubMed: 19036915]

17. Hernan MA, Lanoy E, Costagliola D, Robins JM. Comparison of dynamic treatment regimes via inverse probability weighting. Basic Clin Pharmacol Toxicol. 2006; 98:237-242. [PubMed: 16611197]

18. Macías J, Neukam K, Mallolas J, López-Cortés LF, Cartón JA, Domingo P, et al. Liver toxicity of initial antiretroviral drug regimens including two nucleoside analogs plus one nonnucleoside analog or one ritonavir-boosted protease inhibitor in HIV/HCV-coinfected patients. HIV Clin Trials. 2012; 13:61-69. [PubMed: 22510353]

19. Mocroft A, Kirk O, Gatell J, Reiss P, Gargalianos P, Zilmer K, et al. Chronic renal failure among HIV-1-infected patients. AIDS. 2007; 21:1119-1127. [PubMed: 17502722]

20. Moyer, VA. [6 September 2012] Screening for chronic kidney disease: U.S. Preventive Services Task Force Recommendation Statement.. Ann Intern Med. 20128. http://annals.org/article.aspx? articleID $=1355168$.

21. Rachlis A, Fanning MM. Zidovudine toxicity. Clinical features and management. Drug Saf. 1993; 8:312-320. [PubMed: 8481217]

22. Semba RD, Shah N, Klein RS, Mayer KH, Schuman P, Vlahov D. Prevalence and cumulative incidence of and risk factors for anemia in a multicenter cohort study of human immunodeficiency virus-infected and uninfected women. Clin Infect Dis. 2002; 34:260-266. [PubMed: 11740716]

23. Judy HE, Price NB. Hemoglobin level and red blood cell count findings in normal women. JAMA. 1958; 167:563-566.

24. Haddy TB, Rana SR, Castro O. Benign ethnic neutropenia: what is a normal absolute neutrophil count? J Lab Clin Med. 1999; 133:15-22. [PubMed: 10385477]

25. Crane HM, Grunfeld C, Willig JH, Mugavero MJ, Van Rompaey S, Moore R, et al. Impact of NRTIs on lipid levels among a large HIV-infected cohort initiating antiretroviral therapy in clinical care. AIDS. 2011; 25:185-195. [PubMed: 21150555]

26. Longenecker CT, Scherzer R, Bacchetti P, Lewis CE, Grunfeld C, Shlipak MG. HIV viremia and changes in kidney function. AIDS. 2009; 23:1089-1096. [PubMed: 19352136]

27. O'Donnell EP, Scarsi KK, Darin KM, Gerzenshtein L, Postelnick MJ, Palella FJ. Low incidence of renal impairment observed in tenofovir-treated patients. J Antimicrob Chemother. 2011; 66:11201126. [PubMed: 21393145]

28. Scherzer R, Estrella M, Li Y, Deeks SG, Grunfeld C, Shlipak MG. Association of tenofovir exposure with kidney disease risk in HIV infection. AIDS. 2012; 26:867-875. [PubMed: 22313955]

29. Calza L, Trapani F, Tedeschi S, Piergentili B, Manfredi R, Colangeli V, et al. Tenofovir-induced renal toxicity in 324 HIV-infected, antiretroviral-naïve patients. Scand J Infect Dis. 2011; 43:656660. [PubMed: 21453205]

30. Horberg M, Tang B, Towner W, Silverberg M, Bersoff-Matcha S, Hurley L, et al. Impact of tenofovir on renal function in HIV infected, antiretroviral-naive patients. J Acquir Immune Defic Syndr. 2010; 53:62-69. [PubMed: 19838127]

31. Gallant JE, Moore RD. Renal function with use of a tenofovir-containing initial antiretroviral regimen. AIDS. 2009; 23:1971-1975. [PubMed: 19696652]

32. Davidson MH. Is LDL-C passed its prime? The emerging role of non-HDL, LDL-P, and ApoB in CHD risk assessment. Arterioscler Thromb Vasc Biol. 2008; 28:1582. [PubMed: 18716322]

33. Marks G, Gardner LI, Craw J, Crepaz N. Entry and retention in medical care among HIVdiagnosed persons: a meta-analysis. AIDS. 2010; 24:2665-2678. [PubMed: 20841990] 
34. Radford MJ, Foody JM. How do observational studies expand the evidence base for therapy? JAMA. 2001; 286:1228-1230. [PubMed: 11559269]

35. Marzolini C, Elzi L, Gibbons S, Weber R, Fux C, Furrer H, et al. Prevalence of comedications and effect of potential drug-drug interactions in the Swiss HIV Cohort Study. Antivir Ther. 2010; 15:413-423. [PubMed: 20516560] 


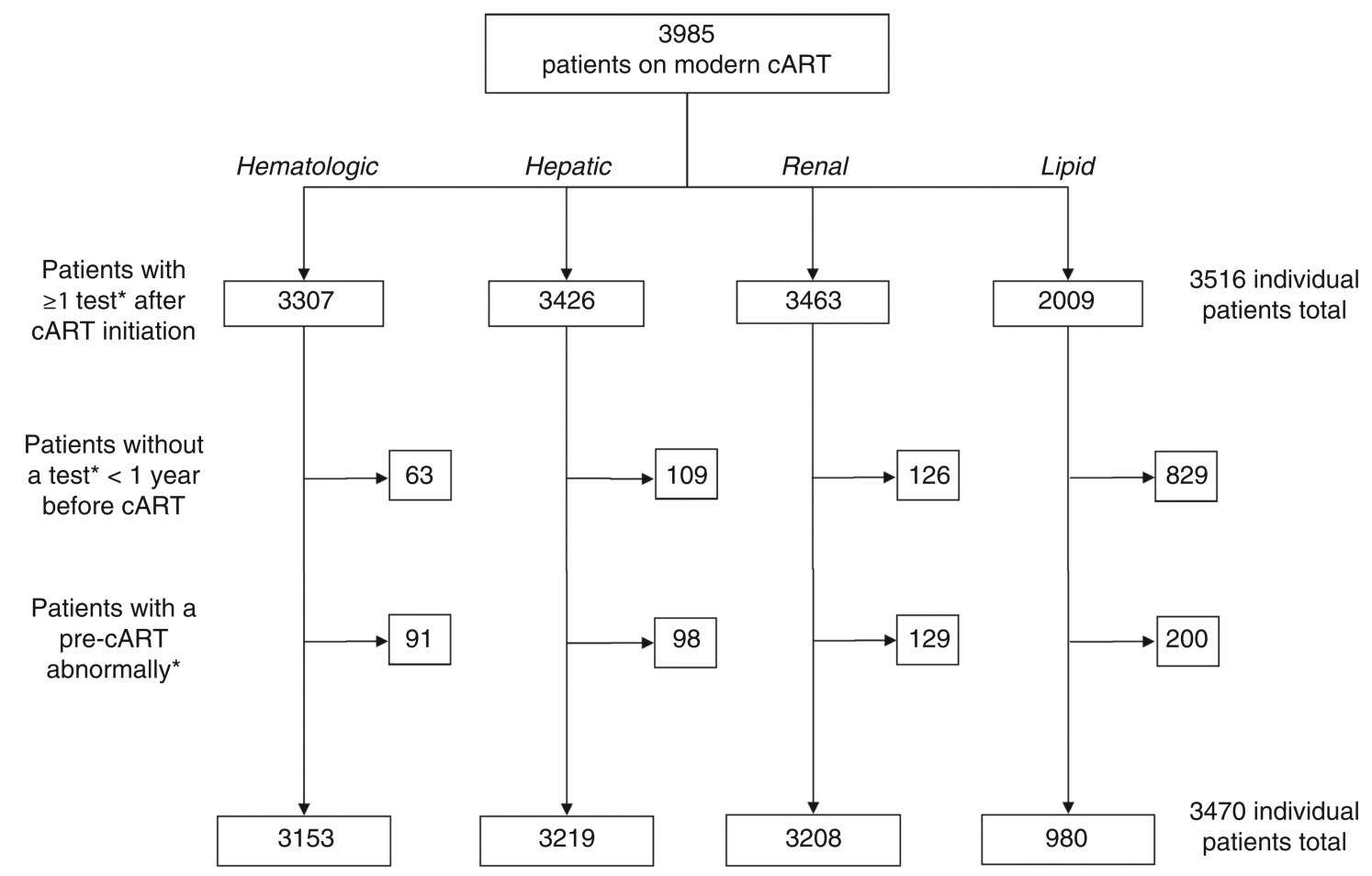

Fig. 1.

Flow chart of exclusion criteria for patient population initiating combination antiretroviral therapy in the Centers for AIDS Research Network of Integrated Clinical Systems, 20002010. *Exclusions only apply to tests for the laboratory type under evaluation. cART, combination antiretroviral therapy. 


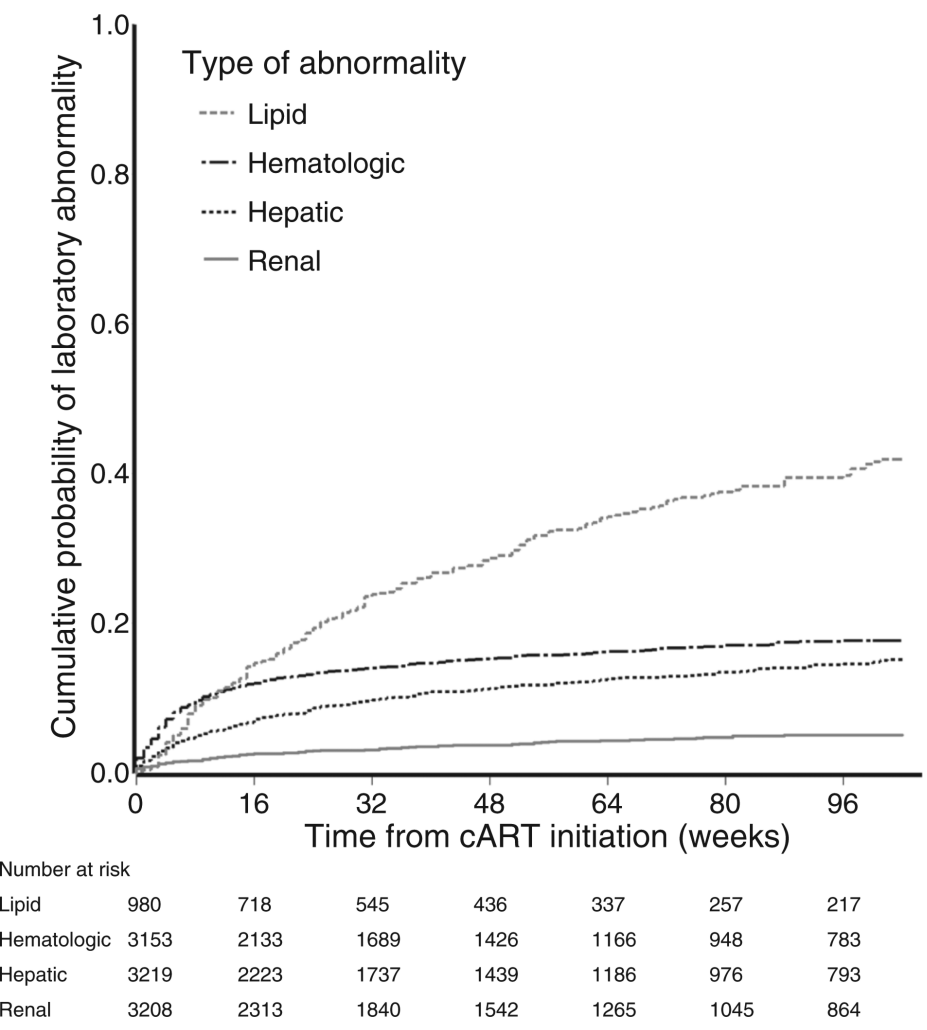

Fig. 2.

Cumulative probability of laboratory abnormality following combination antiretroviral therapy initiation stratified by laboratory type, Centers for AIDS Research Network of Integrated Clinical Systems, 2000-2010. cART, combination antiretroviral therapy. 


\section{Table 1}

Demographic and clinical characteristics of patients initiating combination antiretroviral therapy, Centers for AIDS Research Network of Integrated Clinical Systems 2000-2010.

\begin{tabular}{|c|c|}
\hline Characteristic & $N(\%)$ \\
\hline Total & 3470 \\
\hline \multicolumn{2}{|l|}{ Sex } \\
\hline Male & $2875(82.9)$ \\
\hline Female & $595(17.1)$ \\
\hline \multicolumn{2}{|l|}{ Race/ethnicity } \\
\hline African-American & $1258(36.3)$ \\
\hline Whites & $1520(43.8)$ \\
\hline Other $^{a}$ & $692(19.9)$ \\
\hline $\mathrm{Age}^{b}$ & $40(32-45)$ \\
\hline $\mathrm{CD} 4$ cell count $(\text { cells } / \mu \mathrm{l})^{b}$ & $206(65-316)$ \\
\hline HIV RNA ( $\log _{10}$ copies $\left./ \mathrm{ml}\right)^{b}$ & $4.9(4.4-5.4)$ \\
\hline \multicolumn{2}{|l|}{ cART regimen type } \\
\hline Boosted PI & $1300(37.5)$ \\
\hline Atazanavir & $728(56.0)$ \\
\hline Lopinavir/ritonavir & 467 (35.9) \\
\hline Darunavir & $69(5.3)$ \\
\hline Fosamprenavir & $36(2.8)$ \\
\hline NNRTI & $2164(62.4)$ \\
\hline Efavirenz & $2075(60.0)$ \\
\hline Nevirapine & $89(2.6)$ \\
\hline Other ${ }^{c}$ & $6(0.2)$ \\
\hline Raltegravir & $2(0.1)$ \\
\hline Maraviroc & $4(0.1)$ \\
\hline $\mathrm{ZDV}$ in regimen & 751 (21.6) \\
\hline TDF in regimen & $2518(72.6)$ \\
\hline $\mathrm{ABC}$ in regimen & $357(10.3)$ \\
\hline
\end{tabular}

$\mathrm{ABC}$, abacavir; cART, combination antiretroviral treatment; IQR, interquartile range; NNRTI, nonnucleoside reverse transcriptase inhibitor; PI, protease inhibitor; TDF, tenofovir; ZDV, zidovudine.

${ }^{a}$ Other includes Hispanic, Asian, American-Indian, multiracial, or other race.

${ }^{b}$ Median (IQR) given instead of $N(\%)$.

${ }^{c}$ Other includes integrase inhibitor or entry inhibitor regimens. 


\section{Table 2}

Incident laboratory abnormalities over first 2 years following combination antiretroviral therapy initiation, Centers for AIDS Research Network of Integrated Clinical Systems, 2000-2010.

\begin{tabular}{lcccccc}
\hline & & & & \multicolumn{3}{c}{ IR per 100 person-years (95\% CI) } \\
\cline { 5 - 7 } Laboratory parameter $^{\boldsymbol{a}}$ & Patients & Abnormal results & Person-years & 0-104 weeks & 0-16 weeks & $\mathbf{1 7 - 1 0 4 ~ w e e k s ~}^{\boldsymbol{b}}$ \\
\hline Hematologic & 3153 & 445 & 2930 & $15.2(13.9-16.7)$ & $43.9(39.5-48.9)$ & $4.9(4.0-6.0)$ \\
Hepatic & 3219 & 330 & 3009 & $11.0(9.9-12.2)$ & $23.5(20.4-27.0)$ & $6.2(5.2-7.5)$ \\
Renal & 3208 & 112 & 3175 & $3.5(2.9-4.3)$ & $8.6(6.9-10.9)$ & $1.8(1.3-2.5)$ \\
Lipid & 980 & 291 & 908 & $32.1(28.7-36.1)$ & $48.9(41.1-58.1)$ & $22.7(17.9-28.8)$ \\
\hline
\end{tabular}

CI, confidence interval; HDL, high-density lipoprotein; IR, incidence rate.

${ }^{a}$ Hematologic measures include 14591 hemoglobin tests, 12848 neutrophil tests, and 15207 platelet count tests; hepatic measures include 15128 alanine aminotransferase tests, 15128 aspartate aminotransferase tests, and 7435 direct bilirubin tests; renal measures include 19733 creatinine tests; lipid measures include 632 non-HDL cholesterol tests in a fasting state and 2429 non-HDL cholesterol in a nonfasting state.

${ }^{b}$ Among those with a normal test of that laboratory type within the first 16 weeks. 


\section{Table 3}

Independent predictors of laboratory abnormalities following combination antiretroviral therapy initiation, Centers for AIDS Research Network of Integrated Clinical Systems, 2000-2010.

\begin{tabular}{|c|c|c|c|}
\hline & \multicolumn{3}{|c|}{ aHR $(95 \% \mathrm{CI})$} \\
\hline & 0-104 weeks ${ }^{a}$ & $0-16$ weeks $^{a}$ & $17-104$ weeks $^{a, b}$ \\
\hline \multicolumn{4}{|l|}{ Hematologic } \\
\hline & $N=3153$ & $N=3153$ & $N=1889$ \\
\hline Age (by decade increase) & $1.06(0.94-1.20)$ & $1.04(0.90-1.20)$ & $1.07(0.86-1.34)$ \\
\hline Female sex & $\underline{1.66(1.27-2.16)}$ & $\underline{1.61(1.16-2.22)}$ & $\underline{1.78(1.09-2.92)}$ \\
\hline \multicolumn{4}{|l|}{ Race/ethnicity } \\
\hline Whites & Ref. & Ref. & Ref. \\
\hline African-American & $\underline{1.69(1.28-2.22)}$ & $\underline{1.39(1.00-1.93)}$ & $\underline{2.68(1.59-4.52)}$ \\
\hline Other & $1.35(0.97-1.87)$ & $1.29(0.87-1.91)$ & $1.51(0.81-2.83)$ \\
\hline CD4 cell count (by 100 cells/ $\mu$ increase) & $\underline{0.72(0.66-0.79)}$ & $\underline{0.67(0.59-0.75)}$ & $0.87(0.75-1.01)$ \\
\hline AIDS diagnosis & $\underline{1.38(1.07-1.77)}$ & $\underline{1.56(1.16-2.10)}$ & $1.15(0.70-1.89)$ \\
\hline Boosted PI regimen (compared to NNRTI) ${ }^{c}$ & $\underline{1.27(1.00-1.61)}$ & $1.11(0.83-1.48)$ & $\underline{1.80(1.15-2.81)}$ \\
\hline ZDV use & $\underline{1.78(1.39-2.28)}$ & $\underline{1.84(1.38-2.47)}$ & $1.43(0.86-2.39)$ \\
\hline Hepatitis B & $\underline{1.64(1.06-2.53)}$ & $1.61(0.97-2.69)$ & $1.72(0.75-3.93)$ \\
\hline Hepatitis C & $1.23(0.90-1.68)$ & $1.12(0.77-1.65)$ & $1.50(0.84-2.66)$ \\
\hline Hypertension & $\underline{1.35(1.03-1.76)}$ & $1.27(0.91-1.77)$ & $1.40(0.86-2.26)$ \\
\hline CD4 cell count change (by 50 cells $/ \mu 1$ increase) ${ }^{d}$ & - & - & $\underline{0.89(0.80-0.99)}$ \\
\hline Viral load suppression $(<400 \text { copies } / \mathrm{ml})^{d}$ & - & - & $0.68(0.41-1.12)$ \\
\hline \multicolumn{4}{|l|}{ Hepatic } \\
\hline & $N=3219$ & $N=3219$ & $N=1957$ \\
\hline Age (by decade increase) & $1.01(0.88-1.15)$ & $0.82(0.67-1.01)$ & $\underline{1.24(1.01-1.52)}$ \\
\hline Female sex & $0.94(0.68-1.31)$ & $1.04(0.65-1.67)$ & $0.87(0.52-1.47)$ \\
\hline \multicolumn{4}{|l|}{ Race/ethnicity } \\
\hline Whites & Ref. & Ref. & Ref. \\
\hline African-American & $1.03(0.76-1.41)$ & $1.02(0.64-1.57)$ & $0.88(0.55-1.43)$ \\
\hline Other & $\underline{1.36(1.00-1.84)}$ & $1.26(0.80-1.97)$ & $1.46(0.94-2.29)$ \\
\hline CD4 cell count (by 100 cells/ $\mu$ increase) & $\underline{0.89(0.82-0.97)}$ & $\underline{0.83(0.73-0.94)}$ & $1.02(0.91-1.14)$ \\
\hline AIDS diagnosis & $\underline{1.36(1.03-1.79)}$ & $1.42(0.96-2.11)$ & $1.37(0.89-2.08)$ \\
\hline Boosted PI regimen (compared to NNRTI) ${ }^{c}$ & $\underline{1.70(1.32-2.19)}$ & $\underline{1.64(1.13-2.37)}$ & $\underline{1.75(1.20-2.56)}$ \\
\hline ZDV use & $0.77(0.53-1.11)$ & $0.70(0.42-1.18)$ & $0.92(0.53-1.60)$ \\
\hline Hepatitis B & $\underline{2.88(1.93-4.30)}$ & $\underline{3.39(1.98-5.81)}$ & $\underline{2.34(1.22-4.47)}$ \\
\hline Hepatitis C & $\underline{3.35(2.55-4.41)}$ & $\underline{3.40(2.28-5.05)}$ & $\underline{2.95(1.94-4.48)}$ \\
\hline Hypertension & $1.01(0.75-1.35)$ & $1.06(0.68-1.64)$ & $0.96(0.62-1.49)$ \\
\hline CD4 cell count change (by 50 cells $/ \mu \mathrm{l}$ increase) ${ }^{d}$ & - & - & $0.94(0.86-1.02)$ \\
\hline Viral load suppression $(<400 \text { copies } / \mathrm{ml})^{d}$ & - & - & $0.83(0.51-1.35)$ \\
\hline Renal & & & \\
\hline
\end{tabular}




\begin{tabular}{|c|c|c|c|}
\hline & \multicolumn{3}{|c|}{ aHR $(95 \%$ CI $)$} \\
\hline & $0-104$ weeks $^{a}$ & $0-16$ weeks ${ }^{a}$ & $17-104$ weeks ${ }^{a, b}$ \\
\hline & $N=3208$ & $N=3208$ & $N=2013$ \\
\hline Age (by decade increase) & $\underline{1.86(1.50-2.32)}$ & $\underline{2.02(1.51-2.70)}$ & $1.36(0.96-1.93)$ \\
\hline Female sex & $\underline{1.86(1.13-3.08)}$ & $1.59(0.79-3.22)$ & $\underline{3.08(1.44-6.59)}$ \\
\hline \multicolumn{4}{|l|}{ Race/ethnicity } \\
\hline Whites & Ref. & Ref. & Ref. \\
\hline African-American & $0.76(0.46-1.26)$ & $0.93(0.45-1.93)$ & $0.54(0.25-1.15)$ \\
\hline Other & $0.69(0.36-1.35)$ & $1.12(0.49-2.55)$ & $\underline{0.14(0.03-0.73)}$ \\
\hline CD4 cell count (by 100 cells/ $\mu$ increase) & $\underline{0.77(0.65-0.91)}$ & $\underline{0.70(0.54-0.90)}$ & $\underline{0.77(0.61-0.96)}$ \\
\hline AIDS diagnosis & $1.29(0.79-2.10)$ & $1.84(0.95-3.55)$ & $0.90(0.40-2.04)$ \\
\hline Boosted PI regimen (compared to NNRTI) ${ }^{c}$ & $\underline{1.65(1.06-2.55)}$ & $1.03(0.55-1.92)$ & $\underline{2.12(1.07-4.23)}$ \\
\hline ZDV use & $0.81(0.45-1.46)$ & $0.59(0.24-1.45)$ & $1.06(0.45-2.48)$ \\
\hline Hepatitis B & $0.96(0.33-2.78)$ & $0.87(0.19-3.91)$ & $1.19(0.26-5.54)$ \\
\hline Hepatitis C & $1.21(0.71-2.06)$ & $1.21(0.58-2.50)$ & $1.30(0.55-3.04)$ \\
\hline Hypertension & $\underline{2.31(1.48-3.61)}$ & $\underline{2.25(1.21-4.20)}$ & $\underline{2.81(1.41-5.59)}$ \\
\hline CD4 cell count change (by 50 cells $/ \mu$ increase) ${ }^{d}$ & - & - & $\underline{0.79(0.66-0.93)}$ \\
\hline Viral load suppression $(<400 \text { copies } / \mathrm{ml})^{d}$ & - & - & $0.59(0.28-1.28)$ \\
\hline \multicolumn{4}{|l|}{ Lipid } \\
\hline & $N=980$ & $N=980$ & $N=308$ \\
\hline Age (by decade increase) & $\underline{1.30(1.15-1.47)}$ & $\underline{1.30(1.08-1.57)}$ & $0.99(0.76-1.31)$ \\
\hline Female sex & $\underline{0.64(0.44-0.94)}$ & $0.79(0.44-1.41)$ & $0.60(0.26-1.39)$ \\
\hline \multicolumn{4}{|l|}{ Race/ethnicity } \\
\hline Whites & Ref. & Ref. & Ref. \\
\hline African-American & $\underline{0.69(0.51-0.93)}$ & $\underline{0.54(0.33-0.87)}$ & $0.77(0.40-1.45)$ \\
\hline Other & $1.17(0.86-1.58)$ & $0.95(0.57-1.59)$ & $1.87(0.89-3.93)$ \\
\hline CD4 cell count (by 100 cells/ $\mu 1$ increase) & $1.03(0.96-1.11)$ & $1.09(0.98-1.23)$ & $0.99(0.83-1.18)$ \\
\hline AIDS diagnosis & $\underline{1.63(1.24-2.16)}$ & $1.52(0.96-2.39)$ & $1.64(0.89-3.01)$ \\
\hline Boosted PI regimen (compared to NNRTI) & $1.12(0.87-1.43)$ & $0.96(0.64-1.45)$ & $0.83(0.47-1.47)$ \\
\hline ZDV use & $1.22(0.92-1.62)$ & $1.03(0.65-1.65)$ & $1.30(0.64-2.66)$ \\
\hline Hepatitis B & $\underline{0.36(0.15-0.91)}$ & $0.39(0.09-1.65)$ & $2.08(0.47-9.18)$ \\
\hline Hepatitis C & $\underline{0.36(0.22-0.58)}$ & $\underline{0.38(0.17-0.83)}$ & $1.09(0.46-2.59)$ \\
\hline Hypertension & $\underline{1.42(1.06-1.88)}$ & $1.34(0.86-2.08)$ & $1.89(0.93-3.86)$ \\
\hline CD4 cell count change (by 50 cells/ $\mu 1$ increase) ${ }^{d}$ & - & - & $1.02(0.90-1.14)$ \\
\hline Viral load suppression $(<400 \text { copies } / \mathrm{ml})^{d}$ & - & - & $0.60(0.30-1.22)$ \\
\hline
\end{tabular}

Underlined associations are those with $95 \%$ confidence intervals that do not overlap 1.0. aHR, adjusted hazard ratio; CI, confidence interval; NNRTI, nonnucleoside reverse transcriptase inhibitor; PI, protease inhibitor; ZDV, zidovudine.

${ }^{a}$ All models adjusted for other covariates listed and study site.

${ }^{b}$ Among those with a normal test of that laboratory type within the first 16 weeks.

${ }^{c}$ Associations were different for atazanavir/ritonavir and lopinavir/ritonavir for some of the abnormalities in the first 16 weeks; both boosted PIs were associated with higher incidence of hematologic, hepatic, and renal toxicities after 16 weeks compared to NNRTI use (data not shown). 
${ }^{d}{ }_{\mathrm{CD} 4}$ cell count change was evaluated as the difference between the baseline measure and the most recent measure before week 16. Viral load suppression was evaluated based on most recent measure before week 16. Both of these covariates were only included in regression analysis for predicting laboratory abnormalities after week 16 . 


\section{Table 4}

Rates of laboratory abnormalities between 17 and 104 weeks following combination antiretroviral therapy initiation stratified by select patient demographic and clinical characteristics, Centers for AIDS Research Network of Integrated Clinical Systems, 2000-2010.

\begin{tabular}{lcc}
\hline & \multicolumn{1}{c}{ Incidence rate per 100 person-years (95\% CI) } \\
\cline { 2 - 3 } Abnormality type and subgroup & $\mathbf{0 - 1 6}$ weeks & 17-104 weeks \\
\hline Hepatic total & $23.5(20.4-27.0)$ & $6.2(5.2-7.5)$ \\
Neither hepatitis B or C & $15.9(13.1-19.2)$ & $4.2(3.3-5.2)$ \\
Either hepatitis B or C & $60.1(48.5-74.4)$ & $20.2(15.4-26.5)$ \\
NNRTI-based regimen with neither hepatitis B or C & $12.2(9.6-15.5)$ & $3.2(2.4-4.2)$ \\
PI-based regimen with neither hepatitis B or C & $43.2(32.8-56.8)$ & $6.2(4.6-8.3)$ \\
NNRTI-based regimen with either hepatitis B or C & $22.7(17.9-28.7)$ & $14.4(10.2-20.3)$ \\
PI-based regimen with either hepatitis B or C & $80.3(62.8-103.5)$ & $28.4(20.8-38.7)$ \\
Renal total & $8.6(6.9-10.9)$ & $1.8(1.3-2.5)$ \\
Age <60 years without hypertension & $6.6(4.9-8.9)$ & $1.3(0.8-1.9)$ \\
Age \60 years or with hypertension & $15.8(11.0-22.7)$ & $3.6(2.2-5.8)$ \\
NNRTI-based regimen without hypertension & $5.5(3.8-8.0)$ & $0.9(0.5-1.5)$ \\
PI-based regimen without hypertension & $8.2(5.5-12.1)$ & $1.9(1.1-3.2)$ \\
NNRTI-based regimen with hypertension & $14.3(9.3-21.9)$ & $2.8(1.5-5.0)$ \\
PI-based regimen with hypertension & $21.2(13.7-32.6)$ & $5.9(3.4-10.3)$ \\
\hline
\end{tabular}

CI, confidence interval; IR, incidence rate; NNRTI, nonnucleoside reverse transcriptase inhibitor; PI, protease inhibitor; ZDV, zidovudine. 